\title{
Survey: Big Data Analytics in Agricultural Products Logistics
}

\author{
${ }^{1}$ S.Priyanka, ${ }^{2}$ Dr.A.Prema \\ ${ }^{1}$ Research Scholar, ${ }^{2}$ Associate Professor, Department of Computer Science, Vels Institute of \\ Science, Technology and Advanced Studies (VISTAS), Chennai, India. \\ 1drpriyanka06phd@gmail.com, ${ }^{2}$ unjanai@gmail.com
}

\begin{abstract}
Big Data Analytics is one of the most cutting edge technology in the world. Big Data Analytics will provide data management to store, process and analyze the huge amount of data. Agricultural is one of the domains that assuring big data analytics used to make a change in the field. With the consistent enhancements of the peoples living lifestyle, step by step peoples concentrate on the demand of perishable agricultural products. However, the periodic eruption of food quality and safety issues affected the concern of the end-users. To enrich the distribution performance of agricultural product logistics and to provide the freshness, quality, and safety of the agricultural products has become a thread of the current agricultural domain. Big Data Analytics in Agricultural Products Logistics has an essential prospect of optimizing the distribution path of products, prediction of product demands in the market, traceability of the products, analyzing customer feedbacks and increasing overall performance of logistics in agriculture. This paper investigates the key challenges, methods used, technologies used, algorithms for distribution of products and future of Big Data Analytics in agro-logistics.
\end{abstract}

Keywords -- Big Data, Path Planning Algorithm, Agro Logistics, Big Data Analytics, Agriculture, Supply Chain

\section{INTRODUCTION}

The word 'Agri-Food 4.0' is a resemblance of the term 'Industry 4.0', from the abstraction of 'Agriculture 4.0'. In worldwide, Agri-Food is the multifaceted industry that threatens a wide range of processes, operations, and roles. The 'Industry 4.0' allows an organization to transmit realtime information in terms of behavior and performance. Logistics in Agricultural Product is emerging as a strategic function that denotes the complete value chain [1]. The rising significance of logistics in agriculture can be credited to the fact that each consumer demands a standard supply chain of perishable products with good conditions at exact location at high quality and quantity at a minimal cost. However, the practice of the right methods, approaches, and methodologies for improving Agri product logistics performances is quite challenges. Thus, the concept of 'Industry4.0' augmented and adapted to 'Agriculture 4.0' to analyze the behavior and performance in this specific domain.

\section{BIG DATA}

Big Data is the mammoth volume of information from distinct sources like social media data, business data, sensor data and more. Mainly, big data helps for real-time applications, business and more. By processing the large volume of data using the analytics of big data to scan hidden patterns in the data. Big Data used in Agricultural domain in various fields of pre-harvesting as soil management, weather forecasting, yield prediction, pesticides management, etc., and post-harvesting as supply chain management, logistics, etc.,

Big Data Analytics is the process used to merge business and traditional analytics [2]. In Big Data Analytics, Predictive Analytics is mainly used to identify the future opportunities of data modeling in the high level of advancement and usually incorporated AI and Machine Learning. There is a lot of technique to analyze and predict the data such as classification, clustering algorithm, Association rule mining. It helps govern the field data as well as controlling field activities which bring versatility.

Big Data technologies like Hadoop, HDFS, MapReduce, Pig, Hive, Spark, STORM, H-based, sqoop, Zookeeper and more. The agricultural logistics sector consists of a huge volume and variety of data [3]. It focuses on the Agri logistics field to bring an innovative path to determine design, conceptualization, development, and evaluation [4].

\section{LITERATURE REVIEW}

The number of devices connected to the internet in Agriculture from the processes of pre-harvesting and postharvesting increases the data size and its types which makes the farmers take decision-based on climate, demand 
of the products and to optimize the distribution path and process to deliver the perishable agricultural products to the customers in less period. Big Data Analytics has fabricating innovative demonstration compared to traditional ones. Since the level of food, product wastage is increasing day by day in the world during logistics service. To rectify the food product wastage problem, this paper reviews the algorithm used to optimize the distribution path planning and process of the products on time with good quality.

1. Leng, Kaijun, et al. (2019) [5], integrated and analyzed the efficiency and application of RFID Technology in the production, processing and the detection of the agricultural product supply chain. Agricultural products logistics information systems were introduced.

2. Parvin, Sazia, et al. (2019) [6], This paper proposed architecture for Smart Food Security System using IoT and Big Data Analytics through supply chain management of smart agro-business which use the different sensor to capture real data from the agriculture sector. Developed advanced big data analytics to make a reliable decision based on collected data.

3. Lezoche, Mario, et al. (2020) [7], reviewed the emerging technologies like Big Data, BlockChain, and Artificial Intelligence and supply chain methods and analyzed to view upcoming paths of the agro-food domain.

4. Miranda, Jhonattan, et al. (2019) [8], The 'Sensing, Smart and Sustainable' (S3)' technologies describe how the agro-food sector can be developed using a systematic process for new product development.

5. Belaud, Jean-Pierre, et al. (2019) [9], proposed an approach to validate the wastage of agriculture by using ecological effects to assess various processes. The ByProduct is used in the process of bioconversion of lignocellulosic biomass to produce bioenergy, biomolecules, and biomaterials.

6. Boshkoska, Biljana Mileva, et al. (2019) [10], proposed a Decision Support System (DSS) in two phases. A) Identification of common knowledge boundaries using ML (Machine Learning) and Ontology technologies. B)
To evaluate the existing knowledge boundaries, transforming obtained ontology into DSS.

7. Huai, Chu-Xuan, et al. (2019) [11], proposed to study the vehicle routing problem with multi-type vehicles in the cold chain logistics system by minimizing the cost of both distribution and cargo damage. To solve the problem, a Genetic Algorithm is applied based on two decoding principles as giving the priority to cargo damage and capacity respectively.

8. Yin, Xiao, et al(2017) [12], proposed the CC-Ant Algorithm based on an ant colony algorithm to solve constraint optimization problems as a distribution of cold chain logistics, minimize total cost which includes the cost of vehicles used, transportation cost, customer penalty, and damage cost. Numerical experiments of the proposed algorithm are superior to existing work in both cost and path with less period.

9. Zhao, Guoqing, et al. (2019) [13], reviewed the blockchain technology, main applications and challenges as the integrity of data, avoid tampering, trust, translucence, invariability, full traceability of stored transaction records of all agro-food value chain partners.

10. Gawanmeh, Amjad, et al. (2019) [14], proposed architecture for the security of big data in agricultural supply chain management systems. Improving techniques for capturing, gathering, securing and analyzing big data in the supply chain system using framework as big data aggregation, big data analysis, big data security, and knowledge discovery.

\section{PATH PLANNING ALGORITHMS}

The purpose of path planning algorithm is to determine a shortest path from one location to another location. Path planning algorithms mainly used to solve the problems in different fields from simple spatial route planning to selection of appropriate sequence that is required to achieve a certain goal [15]. In Agricultural Products Logistics, the path planning algorithm types are implemented to optimize the path and process of products distribution. This paper investigates the various types of path planning algorithms.

TABLE 1 EXISTING PATH PLANNING ALGORITHM

\begin{tabular}{|l|l|l|l|}
\hline \multicolumn{1}{|c|}{ Year } & \multicolumn{1}{|c|}{ Author \& Title } & Path Planning Algorithm & Description \\
\hline 2019 & {$[16]$} & Dijkstra Algorithm & $\begin{array}{l}\text { This paper defines, tracking the material flow of agricultural } \\
\text { products using RFID technology is to conduct processing } \\
\text { research through Dijkstra Algorithm. }\end{array}$ \\
\hline 2017 & {$[17]$} & $\begin{array}{l}\text { To estimates, the cost of the logistics process of decay and } \\
\text { illustrates a non-linear programming model for logistics } \\
\text { network configurations for fresh agricultural products using } \\
\text { the Genetic Algorithm. }\end{array}$ \\
\hline 2019 & {$[18]$} & Ant Colony Algorithm & $\begin{array}{l}\text { By optimizing the distribution model, reduce the cost of } \\
\text { logistics based on the ant colony algorithm. }\end{array}$ \\
\hline 2019 & {$[19]$} & $\begin{array}{l}\text { Heuristic Swarm Intelligent } \\
\text { Optimization Algorithm }\end{array}$ & $\begin{array}{l}\text { Proposed to minimize the cost of the distribution process as the } \\
\text { cost of vehicle use, transportation, refrigeration, damage, and } \\
\text { carbon emission. To optimize the distribution path to keep the } \\
\text { freshness of food and reduce energy consumption. }\end{array}$ \\
\hline
\end{tabular}




\begin{tabular}{|l|l|l|l|}
\hline 2019 & {$[20]$} & Traceability Algorithm & $\begin{array}{l}\text { Proposed to integrate blockchain technology and traceability } \\
\text { technology to design traceability algorithms for logistics } \\
\text { service transactions. }\end{array}$ \\
\hline 2019 & {$[21]$} & Seeker Genetic Algorithm & $\begin{array}{l}\text { Proposed a seeker genetic algorithm which is an improved } \\
\text { genetics algorithm to shorten transport distance to minimize } \\
\text { total cost and improve the accuracy of the solution. }\end{array}$ \\
\hline
\end{tabular}

\section{CONCLUSION}

In the agriculture field, agricultural product logistics is one of the main focusing areas to avoid unnecessary wastage of the products before it reaches the end-user. This study deals with various distribution path planning algorithm for agricultural products in logistics. The purpose of those algorithms is to optimize the path planning to deliver the products to end-users on time. The result of this algorithm is limited to give an improved result. Yet, there is a lot of areas to enhance the results to extract the best shortest path for the distribution planning of the products.

\section{REFERENCES}

[1]. http://sohanlal.in/pdfs/Contibution-agri.pdf

[2]. F. Diebold, "Big Data", Pier working paper archive, Penn Institute for Economic Research, 2012.

[3]. D. Laney, "3-D Data Management: Controlling Data Volume, Velocity, and Variety", META Group Research Note 2001.

[4]. Rajeswari, S., K. Suthendran, and K. Rajakumar. "A smart agricultural model by integrating IoT, mobile and cloud-based big data analytics." In 2017 International Conference on Intelligent Computing and Control (I2C2), pp. 1-5. IEEE, 2017.

[5]. Leng, Kaijun, Linbo Jin, Wen Shi, and Inneke Van Nieuwenhuyse. "Research on agricultural products supply chain inspection system based on the internet of things." Cluster Computing 22, no. 4 (2019): 8919-8927.

[6]. Parvin, S., Venkatraman, S., de Souza-Daw, T., Fahd, K., Jackson, J., Kaspi, S., ... \& Gawanmeh, A. (2019). Smart food security system using IoT and big

data analytics. In 16th International Conference on Information Technology-New Generations (ITNG 2019) (pp. 253-258). Springer, Cham.

[7]. Lezoche, M., Hernandez, J., Diaz, M. D. M. A., Panetto, H., \& Kacprzyk, J. (2020). Agri-food 4.0: a survey of the supply chains and technologies for future agriculture. Computers in Industry, 116 .

[8]. Miranda, Jhonattan, Pedro Ponce, Arturo Molina, and Paul Wright. "Sensing, smart and sustainable technologies for AgriFood 4.0." Computers in Industry 108 (2019): 21-36.

[9]. Belaud, Jean-Pierre, Nancy Prioux, Claire Vialle, and Caroline Sablayrolles. "Big data for agri-food 4.0: Application to sustainability management for the by-products supply chain." Computers in Industry 111 (2019): 41-50.

[10]. Boshkoska, Biljana Mileva, Shaofeng Liu, Guoqing Zhao, Alejandro Fernandez, Susana Gamboa, Mariana del Pino, Pascale Zarate, Jorge Hernandez, and Huilan Chen. "A decision support system for evaluation of the knowledge sharing crossing boundaries in agri-food value chains." Computers in Industry 110 (2019): 64-80.
[11]. Huai, Chu-Xuan, Guo-Hua Sun, Ran-Ran Qu, Zhao Gao, and Ze-Hao Zhang. "Vehicle Routing Problem with Multi-type Vehicles in the Cold Chain Logistics System." In 2019 16th International Conference on Service Systems and Service Management (ICSSSM), pp. 1-4. IEEE, 2019.

[12]. Yin, Xiao, Chonglin Gu, Zhenyu Fan, and Hejiao Huang. "Routing Optimization in Distribution of Cold Chain Logistics." In 2017 10th International Symposium on Computational Intelligence and Design (ISCID), vol. 2, pp. 171-176. IEEE, 2017.

[13]. Zhao, Guoqing, Shaofeng Liu, Carmen Lopez, Haiyan Lu, Sebastian Elgueta, Huilan Chen, and Biljana Mileva Boshkoska. "Blockchain technology in agri-food value chain management: A synthesis of applications, challenges, and future research directions." Computers in Industry 109 (2019): 83-99.

[14]. Gawanmeh, Amjad, Sazia Parvin, Sitalakshmi Venkatraman, Tony de Souza-Daw, James Kang, Samuel Kaspi, and Joanna Jackson. "A Framework for Integrating Big Data Security Into Agricultural Supply Chain." In 2019 IEEE Fifth International Conference on Big Data Computing Service and Applications (BigDataService), pp. 191-194. IEEE, 2019.

[15].www.sciencedirect.com/topics/engineering/patp-planning

[16]. Wang, Jianbo, and Jiayue Wang. "Tracking Method of Agricultural Products Logistics Based on RFID Technology." In International Conference on Big Data Analytics for CyberPhysical-Systems, pp. 578-583. Springer, Singapore, 2019.

[17]. Ji, Yingfeng, Hualong Yang, and Meitong Chen. "Logistics network configuration for fresh agricultural products." In 2017 29th Chinese Control And Decision Conference (CCDC), pp. 5724-5727. IEEE, 2017.

[18]. Liu, Lu, Hua Wang, and Shaohua Xing. "Optimization of distribution planning for agricultural products in logistics based on the degree of maturity." Computers and Electronics in Agriculture 160 (2019): 1-7.

[19]. Chen, Limin, Mengli Ma, and Lixin Sun. "Heuristic swarm intelligent optimization algorithm for path planning of agricultural product logistics distribution." Journal of Intelligent \& Fuzzy Systems Preprint (2019): 1-7.

[20]. Liang, Shiaofang, Mingchen Li, and Wenjing Li. "Research on Traceability Algorithm of Logistics Service Transaction Based on Blockchain." In 2019 18th International Symposium on Distributed Computing and Applications for Business Engineering and Science (DCABES), pp. 186-189. IEEE, 2019.

[21]. Zhang, Liyi, Yang Gao, Yunshan Sun, Teng Fei, and Yujing Wang. "Application on Cold Chain Logistics Routing Optimization Based on Improved Genetic Algorithm." Automatic Control and Computer Sciences 53, no. 2 (2019): 169-180. 